\title{
THE PHYSICAL AND PSYCHOLOGICAL MORBIDITY IN MAXILLOFACIAL TRAUMA PATIENTS
}

SW Au Yong L, Ummu Aiman Y and Rahman ZAA.. The physical and psychological morbidity in maxillofacial trauma patients. Annal Dent Univ Malaya 1999; 6: 8 - 10.

\section{ABSTRACT}

The aim of this research is to study how the physical changes in the maxillofacial trauma patients affect them psychologically in patients of different ages, sexes, races, socioeconomic backgrounds, types and severity of injury. The study was conducted by doing a questionnaire survey, which was divided into physical and psychological components. In the physical component, the questions were mainly related to the physical injuries to the patient, which could be visualized clinically and functionally. The psychological component consisted of analyzing the impact of the maxillofacial trauma to the psychological profile. Twenty patients of both sexes and ages, ranged from 15 to 62 years old were interviewed. The main cause of trauma was from motor vehicle accidents. This study appears to show that maxillofacial trauma may have a psychological impact on patients.

Keywords: maxillofacial trauma, physical morbidity, and psychological morbidity

\section{INTRODUCTION}

The face is usually involved in a host of important roles and functions in everyday life. The appearance and 'attractiveness' of a person to other people is partly contributed by the person's face. As a result of maxillofacial trauma, the patient may suffer facial disfigurement, chronic facial pain, anosmia, dysosmia, speech, dental and ophthalmological disabilities. Other than the physical aspect of maxillofacial trauma, another important aspect of maxillofacial trauma that has been overlooked in the dental and surgical clinical scenario is the impact of various injuries in the maxillofacial region on the patient's psychological makeup.

Facial disfigurement is one of the important physical consequence of maxillofacial trauma. Maxillofacial trauma victims often experiences anguish and resentment, and consequently may be emotionally disabled $(1,2)$. Negative reactions from members of public and complaints by the victims of being rejected have been reported in the literature(1). While these negative reactions and the feelings of the rejection may be the result of misperception on the part of the facially disfigured, several studies had suggested that the discrimination that occurred were solely based on appearance and not as a result of conduct and manner (1).

It has also been noted that the interaction between the level of social skill and disfigurement was significantly less influential than the level of social skill displayed (3). Similar conclusions were also reported from burn patients, where the importance of personality and social support rather than the severity of the visible disfigurement were reported (4). It had also been reported that the severity of
SW Au Yong L*, Ummu Aiman $\mathrm{Y}^{*}$ and Rahman ZAA**

*Year V Undergraduate Student (1999/2000)

Faculty of Dentistry, University of Malaya

**Associate Professor

Department of Oral and Maxillofacial Surgery

Faculty of Dentistry, University of Malaya

**Name and address of author to whom requests for reprints should be addressed.

a deformity was inversely related to the psychological impact of the patient (5).

Another important physical component of maxillofacial trauma is chronic facial pain. The face and scalp have a rich sensory innervation, chiefly from branches of the trigeminal nerve, and nerve injury is a common complication of craniomaxillofacial trauma. Victims of nerve injury may complain of numbness, abnormal sensation and discomfort but very few further complained of chronic pain. The formation of a traumatic neuroma was often postulated, and in some cases, this appeared to be the cause of pain(1).

Anosmia and dysosmia may also occur after maxillofacial trauma. Both disabilities result from damage to the olfactory nerves which is a very important and often neglected component of many craniomaxillofacial injuries. If the natural protection via sense of smell was destroyed, this may lead to failure to smell burning or escaping gas, which further endanger the patient, while also depriving the patient from the pleasure of eating (1).

Speech disabilities after maxillofacial trauma may develop in several ways. These disabilities were dependent on the site of injury, whether there was cerebral injury, damage to the muscles of articulation, nasal obstruction, or impaired oral or labial sensation (1).

Dental disabilities due to maxillofacial trauma may affect a person functionally as well as aesthetically. Patients might not be able to afford complex and expensive aesthetic restorative treatment. Provision of an acrylic partial denture might then change taste perception and alter eating habits.

Ophthalmological disability such as eye disfigurement, visual loss and diplopia would have significant effects on the victim's interaction with other people. These may result from disturbances of the shape of the globe and the eyelids and/or disturbances in the colour, the clarity and the position of the eye (1).

Many patients with orofacial trauma have no other injuries, whereas others have multiple injuries involving several physiological and anatomical systems. Furthermore, some patients with minor injuries may suffer 
severe psychological problems while some severely injured patients may have only minor psychological problems(6). It is therefore necessary that both the overall psychological impacts of an incident and the specific psychological problem associated with orofacial injury be taken into account when managing patients with maxillofacial trauma(7). Thus, the objectives of this study were to evaluate the physical and psychological morbidity of the maxillofacial trauma patients. Another objective was to discern and compare the patients' responses to maxillofacial trauma in relation to their age, sex, race, socioeconomic background and the types and severity of injury.

\section{MATERIALS AND METHOD}

This study consisted of a cross-sectional overview of patients with maxillofacial trauma seeking/referred for treatment in the Oral and Maxillofacial Surgery Department (OMFS), Faculty of Dentistry, University of Malaya in a period of 4 weeks in March 1999. All patients regardless of age, sex, race, type and cause of injury and stage of treatment were included in this 4 weeks' survey.

A survey form was devised based on selected questions found in existing survey forms, which were taken from the Brief Disaster Questionnaire (International Liaison Group) (6), and the Late Effect of Accidental Injury Questionnaire (7). These selected questions were modified to reflect the objectives of this study.

The survey form was divided into three parts: the background information, the physical and psychological portions. The background information included the recording of the age, sex, occupation of the patient; the time period from the occurrence of the injury to the time of the interview; the type and cause of injuries and the type of treatment received.

The physical portion involved recording the clinical observation of the patients' face and mouth, with radiological imaging when appropriate. These include the severity of the injuries suffered by the patients'. Severity of the injuries was divided into three categories namely, mild, moderate and severe. The mild injury was defined as those with no obvious deformity, disfigurement, disability or dysfunction to the face requiring either elective or conservative treatment only. Those in the moderate category would have some form of deformity, disfigurement, disability or dysfunction to the face while those with obvious deformity, disfigurement, disability or dysfunction to the face requiring surgical correction were placed into the severe category.

In the psychological portion, the patients were asked to gauge the level of their self-consciousness towards their disfigurement. These included their perception and experience of how other people react to their appearance, how the actual incident and the injury? affected them psychologically and also if there was any change in their social interaction brought about by the sequelae of the injury.

\section{RESULTS}

During the one-month period, twenty maxillofacial trauma patients attended the OMFS Clinic. There were 11 Malays,
4 Chinese, 3 Indians, 2 foreign (1 Indonesian and 1 Bangladeshi) patients. They consisted of 15 males and 5 females whose age ranges from 15 to 62 years old. Eighty percent of these patients were between 20 to 30 years of age.

The distribution of the time period from the occurrence of the injury to the time of the interview of the patients was varied. The time period ranged from 14 hours to 19 months after the associated accidents. At the time of the interview, $55 \%$ of the patients were discharged from the wards/outpatients, $25 \%$ were on medical leave and only $20 \%$ were still in the ward.

The most common cause of maxillofacial trauma in this study was due to motor vehicle accidents (MVAs), totaling $70 \%$. Other causes included occupation-related injuries $(10 \%)$, domestic injuries $(10 \%)$, sport-related accidents $(5 \%)$ and assault injuries $(5 \%)$.

Half of the patients in this study who were all males, suffered permanent physical changes. All 3 categories of severity of physical changes were recorded. The mild category recorded was $30 \%$, moderate $40 \%$ and the severe category $30 \%$ of all cases. These changes were dental injuries, asymmetry of the nose and blindness. The dental injuries were crown and root fractures, and tooth avulsion.

Many sites in the craniofacial region were susceptible to trauma. The most susceptible region to trauma was the mandible, most notably fractures of the body of the mandible, which occurred up to $26 \%$ of the cases seen(Table 1). This was followed by injuries to the maxillary complex (18\%), dental injuries (18\%) and soft tissue injuries (32\%).

Ninety percent(17) of the patients claimed that there were reduced physical function such as eating, speaking and other physical activities. Out of these cases, $85 \%$ faced difficulties in eating due to tooth loss and fractures, displaced jaws and malocclusion. Sixty five percent(13) of the patients reported that their speech was affected while fifteen percent(3) claimed reduced physical activity due to the maxillofacial trauma.

For the psychological component, $50 \%$ of all patients felt self-conscious of their facial disfigurement and $25 \%$ of them tried to conceal their disfigurement. Ten percent of the patients felt extremely sad/depressed, $25 \%$ very sad/depressed, $35 \%$ felt a little sad/depressed while $30 \%$ did not feel sad/depressed after the trauma. In most of the cases evaluated, the patients did not have a problem going out in public.

Table 1 : Location and severity of maxillofacial trauma suffered by the patients.

\begin{tabular}{|c|c|c|c|c|}
\hline & Mild & Moderate & Severe & Total \\
\hline & $\mathrm{No}(\%)$ & $40.8 \%$ & No. $(\%)$ & $\mathrm{No}(\pi / 0)$ \\
\hline Mandible & $3=(30)^{2}$ & $2(20)$ & $5(50)$ & $10(26)$ \\
\hline Maxilla & $0(0)$ & $1 \quad(14)$ & $6(86)$ & $7(18)$ \\
\hline Dental & 3 (43) & 2 (29) & $2(29)$ & $7(18)$ \\
\hline Eyes & $0 \quad(0)$ & $0 \quad(0)$ & $1 \quad(100)$ & $1(3)$ \\
\hline of skull & $0(0)$ & $0 \quad(0)$ & $1 \quad(100)$ & $1(3)$ \\
\hline Sof & $1,(-8)$ & $5 \quad(42)$ & $6(50)$ & $12(32)$ \\
\hline & $7(18)$ & $150(26)$ & $21(55)$ & $38(100)$ \\
\hline
\end{tabular}




\section{DISCUSSION}

Motor vehicle-related accidents were the number one cause of maxillofacial trauma in our study. The region that bears the brunt of the trauma was usually the mandible.

In most cases the women were self-conscious in comparison to the men, even though their injuries were much less severe than the men. In this study, only one quarter of the patients was females. They were all between 20 to 30 years old and were single. They only incurred mild to moderate injuries with no permanent physical changes. Nonetheless, three of them felt self-conscious of their appearance, although they suffered no obvious facial deformity. Whereas in comparison, only six men out of the fifteen did, and they all suffered obvious deformity. This could be attributed to the fact that women were more preoccupied with their appearance (8).

There were trends in this study that would suggest a relation between physical and psychological morbidity due to maxillofacial trauma where $50 \%$ of all patients felt self conscious of their facial disfigurement arising from the maxillofacial trauma. However, only $10 \%$ of patients felt very sad or depressed while $35 \%$ and $30 \%$ felt a little sad and no feeling respectively. Raphael et al (1989)(6) reported that these feelings were apparent within a week or two of the injuries. These feelings were probably due to the pain and discomfort, the changed appearance, the reduced function and the anxiety suffered by the patients.

Feelings of sadness or depression did not seem to depend on the severity of the injuries suffered. These feelings tend to decrease with time, which could be due to the reduction in active sensation and discomfort. The patients reportedly feels comforted in the presence of other people especially their loved ones, denoting the need for psychological support. These findings concur with other studies that remarked that personality traits affect the adjustment of the patients (9).

However the limited sample in this study does not permit a statistical evaluation of the observations from this study. Thus, this study was unable to dwell deeper into the significance of both the physical and psychological morbidity suffered by the patients. The injuries of differing nature, areas and severity made data comparison and correlation difficult. Other factors such as injuries to other parts of the body would also contribute to the changes in the quality of life, making it difficult to conclude that the psychological response was due to the craniofacial region. The wide distribution in the time periods of occurrence of injury to the time of interview further made data comparison and correlation difficult.

It was also not possible to compare the patients' responses to maxillofacial trauma with regards to age, sex, race, socioeconomic background and the types and severity of injury due to the small number of patients in this study. Thus, future studies of similar nature requires the involvement of a bigger sample and interviews with the patient should be conducted at the same time period after the trauma.

\section{CONCLUSION}

In conclusion, this study appears to show that maxillofacial trauma may have a psychological impact on the patients. Arising from this, it is thus important to understand the impact of the maxillofacial trauma to an individual, physically and psychologically.

Even as busy clinicians, time and effort must be given to manage these trauma patients with sympathy and empathy. There is no use mending the patients physically, but at same time, neglecting their need for psychological support, for both the physical and psychological components are inseparable.

\section{ACKNOWLEDGEMENTS}

This paper was part of a presentation at the 'First Dental Student Scientific Conference' for the Dental Faculty, University of Malaya held on 19 - 20 November 1999. The authors wished to thank the Dean of the Dental Faculty for approving the fund required for this study. The authors also wished to thank Ms. Vivien Tuan Hui-wearn and Ms. Nor Azwa Hashim for their assistance.

\section{REFERENCES}

1. Wood M, Hammerton M. Impairments and disabilities. In David DJ, Simpson DA ed Craniomaxillofacial trauma. Churchill Livingstone 1995: 649-75.

2. Macgregor FC. Facial disfigurement: Problems and management of social interaction and implications for mental health. Aesthet Plast Surg 1990; 14: 249-257.

3. Rumsey N, Bull R. The effects of facial disfigurement on social interaction. Hum Learn J Prac Res Applens 1986; 5: 203-208.

4. Hill C. Psychosocial adjustment of adult burn patients: is it more difficult for people with visible scars? $\mathrm{Br} \mathrm{J}$ Occupat Ther 1985; 48: 281-283.

5. Lansdown R, Lloyd J, Hunter J. Facial deformity in childhood: severity and psychological adjustment. Child Care Health Dev 1991; 17: 165-171.

6. Raphael B, Lundin T, Weisaeth L. A research method for the study of psychological and psychiatric aspects of a disaster. Acta Psychiatr. Scand 1989 (suppl); 80: 1 , no. 353, 1-75.

7. Malt UF, Myhrer T, Blikra G, et al. Psychopathology and accidental injuries. Acta Psychiatr. Scand 1987; 76: 261.

8. Kleck RE, Strenta AC. Gender and responses to disfigurement in self and others. J Soc Clin Psychol 1985; 3: 257-267.

9. Shepherd JP. Strategies for the study of long-term sequelae of oral and facial injuries. J Oral Maxillofac Surg 1992; 50: 390-399. 